\title{
THE COMPACT OPERATORS ARE NOT COMPLEMENTED IN $\mathscr{B}(\mathscr{H})$
}

\author{
JOHN B. CONWAY
}

\begin{abstract}
If $\mathscr{H}$ is an infinite dimensional Hilbert space then it is shown that the space of compact operators is not complemented in the space of all bounded operators.
\end{abstract}

The purpose of this note is to prove a Theorem which most of my colleagues "know" without ever having seen or heard of a proof. The statement is as follows.

THEOREM. If $\mathscr{H}$ is an infinite dimensional Hilbert space and $\mathscr{C}$ is the space of all compact operators on $\mathscr{H}$ then there is no bounded projection of $\mathscr{B}(\mathscr{H})$ (the space of all bounded operators on $\mathscr{H})$ onto $\mathscr{C}$.

More general theorems can be found in [1] and [2].

To prove this we first introduce the following notation. For any set $I$ let $l^{\infty}=l^{\infty}(I)$ be all bounded functions from $I$ into $C$; and let $c_{0}=c_{0}(I)$ be all functions on $I$ which vanish at infinity (i.e. for each $\varepsilon>0$ the set of points $i \in I$ for which $|f(i)| \geqq \varepsilon$ is finite). We use the fact that there is no bounded projection of $l^{\infty}$ onto $c_{0}$. All my colleagues seem to have heard of or seen a proof of this fact, although too few have seen the proof due to R. Whitley [3].

Let $\left\{e_{i}: i \in I\right\}$ be an orthonormal basis for $\mathscr{H}$ and for each index $i \in I$ let $P_{i}$ be the orthogonal projection of $\mathscr{H}$ onto the one-dimensional span of $e_{i}$. If $f$ is an element of $l^{\infty}$ then

$$
\rho(f)=\sum_{i \in I} f(i) P_{i}
$$

defines a bounded operator on $\mathscr{H}$ (this sum is the limit in the weak operator topology of the net of all finite partial sums). It follows that $\rho$ is an isometry of $l^{\infty}$ into $\mathscr{B}(\mathscr{H})$ and

(1) $\rho\left(c_{0}\right) \subset \mathscr{C}$.

Received by the editors November 23, 1970.

AMS 1970 subject classifications. Primary 46C10, 47D15.

Key words and phrases. Hilbert space, bounded operators, compact operators, noncomplemented subspace.

(c) American Mathematical Society 1972 
Now define $\sigma: \mathscr{B}(\mathscr{H}) \rightarrow l^{\infty}$ by $\sigma(T)(i)=\left(T e_{i}, e_{i}\right)$ for each $T \in \mathscr{B}(\mathscr{H})$ and $i \in I$. Again $\sigma$ is a linear map and $\|\sigma(T)\| \leqq\|T\|$. Also

(2) $\sigma(\mathscr{C})=c_{0}$, and

(3) $\sigma(\rho(f))=f$ for each $f$ in $l^{\infty}$.

So if $\pi$ is a bounded projection of $\mathscr{B}(\mathscr{H})$ onto $\mathscr{C}, \sigma \circ \pi \circ \rho$ is a bounded projection of $l^{\infty}$ onto $c_{0}$. Thus, no such map $\pi$ can exist.

Notice that this also says that there is no linear lifting of the Calkin algebra into $\mathscr{B}(\mathscr{H})$.

\section{REFERENCES}

1. E. O. Thorp, Projections onto the subspace of compact operators, Pacific J. Math. 10 (1960), 693-696. MR 22 \#4955.

2. D. Arterburn and R. Whitley, Projections in the space of bounded linear operators, Pacific J. Math. 15 (1965), 739-746. MR 32 \#4507.

3. R. Whitley, Projecting $m$ onto $c_{0}$, Amer. Math. Monthly 73 (1966), 285-286.

Department of Mathematics, Indiana University, Bloomington, Indiana 47401 